\title{
Coupled Fixed Point Theorems in Vector b-metric Space
}

\author{
Mamta Kamra, Rahul Hooda, Archana Malik
}

\begin{abstract}
This paper consists of some coupled and common coupled fixed point theorems in vector b-metric spaces. Vector b-metric space or E-b-metric space was introduced by Petre [6] merging the concepts of vector metric space as introduced by Cevik [4] and b-metric space as introduced by Czerwik [5]. We generalize the results of Shatnanawi and Hani [8] and Rao et al. [7].
\end{abstract}

Keywords: Coupled Fixed, b-metric Space.

\section{INTRODUCTION}

The notion of coupled fixed point for metric spaces was initiated by Bhaskar and Lakshmikantan [12]. Thereafter several authors investigated coupled fixed point theorems for various general metric spaces [12-14]. In 1989, Bakhtin [3] introduced the concept of b-metric space. Chao et al. [13] and Lakshikanthan [14] have probed coupled fixed point theorems on b-metric space. Cevik and Altun [4] introduced the concept of vector metric space and proved some fixed point theorems on this space. Petre [6] defined the vector b-metric space or E-b-metric space. We prove some results related to coupled fixed points on E-b-metric space.

\section{PRELIMINARIES}

We present here various definitions and results that will be used in the sequel.. For definitions and results related to Riesz space, we refer Aliprantis and Border [2] and that for vector metric spaces, one can see Cevik and Altun [4].

Definition 2.1 A set $\mathrm{Z}$ with binary relation $(\leq)$ which is reflexive, antisymmetric and transitive is called partial

A poset $(Z, \leq)$ is said to be linearly ordered or totally ordered or chain if for each pair $u, v \in Z$, we have either $u \leq v$ or $v \leq u$.

Definition 2.2 A poset in which every subset with two elements has a supremum or an infimum is called lattice. A lattice in $\mathrm{Z}$ is said to be complete if every subset has a supremum or infimum. A lattice is Dedekind complete if every non empty subset of a lattice which is bounded below (above) has a infimum (supremum).

Revised Manuscript Received on November 22, 2019.

* Correspondence Author

Mamta Kamra, Department of Mathematics, Indira Gandhi university, Meerpur(Rewari)-122502Haryana, India.

Rahul Hooda, Department of Mathematics, Maharshi Dayanand University, Rohtak-124001 Haryana, India University, Rohtak-124001 Haryana, India ordered set(poset).

Archana Malik, Department of Mathematics, Maharshi Dayanand

Definition 2.3 A partially ordered vector space is poset $(E, \leq)$ where $\mathrm{E}$ is a real vector space such that for all $u, v, w \in E$ and $\lambda>0$
(i) $u \leq v$
$\Rightarrow u+w \leq v+w$
(ii) $u \leq v$
$\Rightarrow \lambda u \leq \lambda v$

Definition 2.4 A partially ordered vector space which is also a lattice under its ordering is called a Riesz space.

Notation In a Riesz space, for a decreasing sequence $\left\{u_{n}\right\}$ whose inf $u_{n}=u$. We use the notation $u_{n} \downarrow u$.

Definition 2.5 An Archimedean Riesz space E is a Riesz space in which $\frac{1}{n} u \downarrow 0$ for every $u \in E_{+}$, where $E_{+}=\{u \in E: u \geq 0\}$.

Definition 2.6 In a Riesz space E, A sequence $\left\{u_{n}\right\}$ is order convergent to $u$ written as $u_{n} \rightarrow u$, if there exists a sequence $\left\{a_{n}\right\}$ in E satisfying $a_{n} \downarrow 0$ and $\left|u_{n}-u\right| \leq a_{n}$ for all $n$, where $|u|=u \vee-u$.

The sequence $\left\{u_{n}\right\}$ in a Riesz space $\mathrm{E}$ is order-Cauchy if there exists a sequence $\left\{a_{n}\right\}$ in E satisfying $a_{n} \downarrow 0$ and $\left|u_{n}-u_{n+m}\right| \leq a_{n}$ forall $n$ and forall $m$.

Lemma 2.7 [15] In a Riesz space $\mathrm{E}$, if $u \leq k u$, where $u \in E_{+}, k \in[0,1)$ and $E_{+}=\{u \in E: u \geq 0\}$, then $u=0$.

Example 2.8 $\square^{2}$ is a Riesz space with coordinate wise ordering defined by $\left(u_{1}, u_{2}\right) \leq\left(v_{1}, v_{2}\right) \quad$ iff $u_{1} \leq v_{1}, u_{2} \leq v_{2}$ for all $\left(u_{1}, u_{2}\right),\left(v_{1}, v_{2}\right) \in \square^{2}$.

Definition 2.9 A function $d: Z \times Z \rightarrow E$, where $\mathrm{Z}$ is nonempty set and $E$ is Riesz space is called a E-metric(vector metric) on $\mathrm{Z}$ if it satisfies the following properties :

(i) $d\left(z_{1}, z_{2}\right)=0$ iff $z_{1}=z_{2}$

(ii) $d\left(z_{1}, z_{2}\right) \leq d\left(z_{1}, z_{3}\right)+d\left(z_{3}, z_{2}\right) \quad \forall z_{1}, z_{2}, z_{3} \in Z$

Then triplet $(Z, d, E)$ is said to be vector metric space.

For any $z_{1}, z_{2}, z_{3}, z_{4}$ in a vector metric space, some inequalities listed below are trivial

$$
\text { (a) } 0 \leq d\left(z_{1}, z_{2}\right)
$$



(b) $d\left(z_{1}, z_{2}\right)=d\left(z_{2}, z_{1}\right)$
(c) $\left|d\left(z_{1}, z_{3}\right)-d\left(z_{2}, z_{3}\right)\right| \leq d\left(z_{1}, z_{2}\right)$
(d) $\left|d\left(z_{1}, z_{3}\right)-d\left(z_{2}, z_{4}\right)\right| \leq d\left(z_{1}, z_{2}\right)+d\left(z_{3}, z_{4}\right)$.

Example 2.10 Every Riesz space $E$ is a vector metric space with $d\left(z_{1}, z_{2}\right)=\left|z_{1}-z_{2}\right|$ for all $z_{1}, z_{2} \in E$.

Example 2.11 Let $d: \square \times \square \rightarrow \square^{2}$ be defined as $\quad d(x, y)=\left(\alpha_{1}|x-y|, \alpha_{2}|x-y|\right) \quad, \quad$ where $\alpha_{1}, \alpha_{2} \geq 0$ and $\alpha_{1}+\alpha_{2}>0$. Then $\mathrm{d}$ is a vector metric with $\left(\square, d, \square^{2}\right)$ is a vector metric space.

Example 2.12 $\square^{n}$ is a Riesz space corresponds to partial order defined by

$$
\left(u_{1}, u_{2}, \ldots, u_{n}\right) \leq\left(v_{1}, v_{2}, \ldots, v_{n}\right) \text { if and only if }
$$

$u_{1} \leq v_{1}, u_{2} \leq v_{2}, \ldots, u_{n} \leq v_{n}$.

Define $d: \square^{n} \times \square^{n} \rightarrow \square$ by

$$
d\left(\left(u_{1}, u_{2}, \ldots, u_{n}\right),\left(v_{1}, v_{2}, \ldots, v_{n}\right)\right)=\left(\alpha_{1}\left|u_{1}-v_{1}\right|, \alpha_{2}\left|u_{2}-v_{2}\right|, \ldots, \alpha_{n}\left|u_{n}-v_{n}\right|\right)
$$

where $\alpha_{i}, i \leq 1 \leq n$, are non-negative real numbers with $\alpha_{1}+\alpha_{2}+\ldots .+\alpha_{n}>0$. Then $\left(\square^{n}, d, \square^{n}\right)$ is a vector metric space.

Definition 2.13 Suppose $(Z, d, E)$ is a vector metric space. A sequence $\left\langle z_{n}\right\rangle$ in $\mathrm{Z}$ is said to be E-converges(or vectorial converges) to some $z \in Z$, written as $z_{n} \stackrel{d, E}{\rightarrow} z$, if there exists a sequence $\left\langle a_{n}\right\rangle$ in E such that $a_{n} \downarrow 0$ and $d\left(z_{n}, z\right) \leq a_{n}$ for all $\mathrm{n}$.

Definition 2.14 A sequence $\left\langle z_{n}\right\rangle$ in a vector metric space $(Z, d, E)$ is said to be E-Cauchy if if there exists a sequence $\left\langle a_{n}\right\rangle$ in E such that $a_{n} \downarrow 0$ and $d\left(z_{n}, z_{n+m}\right) \leq a_{n} \forall \mathrm{n}$ and $\mathrm{m}$.

Definition 2.15 Let $Y$ be any subset of a vector metric space Z. Y is said to be E-closed if for every sequence $\left\{z_{n}\right\} \subseteq Y$ and $z_{n} \stackrel{d, E}{\rightarrow} z$, imply $z \in Y$.

It is easy to see that if $z_{n} \stackrel{d, E}{\rightarrow} z$, then the limit of the sequence $z_{n}$ is unique and every subsequence of $\left\langle z_{n}\right\rangle$ E-converges to z. If $y_{n} \stackrel{d, E}{\rightarrow} y$, then $d\left(z_{n}, y_{n}\right) \stackrel{0}{\rightarrow} d(z, y)$.

The concepts of convergence in metric similar to vectorial convergence when $E=\square$. Also, If $\mathrm{d}$ is the absolute valued metric and $Z=E$ then concepts of convergence in order and vectorial convergence coincide.

Definition 2.16 An E-complete vector metric space $\mathrm{Z}$ is a vector metric space in which every E-Cauchy sequence in $Z$ E-converges to a limit in $\mathrm{Z}$. coordinatewise or lexicographical ordering and

Definition $\quad 2.17 \quad[16] \quad A \quad$ mapping $f:(Z, d, E) \rightarrow\left(Y, d^{\prime}, F\right)$ is vectorially continuous at $\mathrm{z}$ if $z_{n} \stackrel{d, E}{\rightarrow} z$ in $\mathrm{Z}$ implies $f\left(z_{n}\right) \stackrel{d^{\prime}, F}{\rightarrow} f(z)$ in $\mathrm{Y}$ and the function $f$ is vectorially continuous on $\mathrm{Z}$ if it is vectorically continuous at each element of $\mathrm{Z}$.

Definition 2.18 [6]A function $d: Z \times Z \rightarrow E_{+}$, where $\mathrm{E}$ is Riesz space and $\mathrm{Z}$ is nonempty set, is said to be E-b-metric if, for any $z_{1}, z_{2}, z_{3} \in Z$ and $s \geq 1$ any real number, the following conditions are satisfied:

(i) $d\left(z_{1}, z_{2}\right) \leq s\left[d\left(z_{1}, z_{3}\right)+d\left(z_{2}, z_{3}\right)\right]$

(i) $d\left(z_{1}, z_{2}\right)=0$ if and only if $z_{1}=z_{2}$.

The triple $(Z, d, E)$ is said to be E-b-metric space.

Example 2.19 Let $Z=L^{p}[0,1]$ with $0<p<1$ and $E=\square^{2}$. Let $d: L^{p}[0,1] \times L^{p}[0,1] \rightarrow \square_{+}^{2}$ be defined by

$$
d\left(f_{1}, f_{2}\right)=\left(\alpha\left\|f_{1}-f_{2}\right\|_{p}, \beta\left\|f_{1}-f_{2}\right\|_{p}\right)
$$

where $\alpha, \beta \geq 0$ and $\alpha+\beta>0$. Then we can deduce that

$$
d\left(f_{1}, f_{2}\right) \leq 2^{\frac{1}{p}}\left[d\left(f_{1}, f_{2}\right)+d\left(f_{2}, f_{3}\right)\right] \text {. }
$$

Hence $\left(Z, d, \square^{2}\right)$ is E-b-metric space with parameter $s=2^{\frac{1}{p}}>1$.

Example 2.20 Suppose $0<p<1, Z=l_{p}$, and $d: l_{p} \times l_{p} \rightarrow \square_{+}^{2}$ is defined as

$$
d(u, v)=\left(\alpha\|u-v\|_{p}, \beta\|u-v\|_{p}\right),
$$

where $\alpha, \beta \geq 0$ and $\alpha+\beta>0$, then $\left(Z, d, \square^{2}\right)$ is E-b-metric space with parameter $s=2^{\frac{1}{p}}>1$.

Definition 2.21 Let $Z=C[-1,1]=E \quad$ and $d: Z \times Z \rightarrow E_{+}$be defined as

$$
d\left(f_{1}, f_{2}\right)=\left(f_{1}-f_{2}\right)^{p}, p>1 .
$$

Then $(Z, d, E)$ is E-b-metric space with parameter $s=2^{\frac{1}{p}}>1$. Since the function $x^{p}(p>1)$ is convex, we have

$$
\left(\frac{1}{2} x+\frac{1}{2} y\right)^{p} \leq \frac{1}{2} x^{p}+\frac{1}{2} y^{p}
$$

so that $(x+y)^{p} \leq 2^{p-1}\left(x^{p}+y^{p}\right)$

Therefore 


$$
\begin{aligned}
d\left(f_{1}, f_{3}\right) & =\left(f_{1}-f_{3}\right)^{p}=\left(f_{1}-f_{2}+f_{2}-f_{3}\right)^{p} \\
\leq & 2^{p-1}\left[\left(f_{1}-f_{2}\right)^{p}+\left(f_{2}-f_{3}\right)^{p}\right] \\
= & 2^{p-1}\left[d\left(f_{1}, f_{2}\right)+d\left(f_{2}, f_{3}\right)\right]
\end{aligned}
$$

Thus the relaxed triangular inequality holds with $s=2^{\frac{1}{p}}>1$

Example $\quad 2.22 \quad$ Let $\quad Z=\square^{2}, E=\square^{2} \quad$ and $d: Z \times Z \rightarrow \square^{2}$ be defined as

$$
d\left(\left(u_{1}, v_{1}\right),\left(u_{2}, v_{2}\right)\right)=\left(\alpha\left|u_{1}-u_{2}\right|^{2}, \beta\left|v_{1}-v_{2}\right|^{2}\right) .
$$

where $\alpha, \beta \geq 0$ and $\alpha+\beta>0$, then $\left(Z, d, \square^{2}\right)$ is E-b-metric space with parameter $s=2>1$.

Example 2.23 Let $Z=\{0,1,2\} \quad, \quad E=\square^{2}$ and $d: Z \times Z \rightarrow \square^{2}$ be defined as

$$
\begin{aligned}
& d(0,1)=d(1,0)=(1,1) \\
& d(1,2)=d(2,1)=(1,1) \\
& d(0,2)=d(2,0)=(4,4)
\end{aligned}
$$

Since $d(0,2)=(4,4)\rfloor d(0,1)+d(1,2),(Z, d, E)$ is E-b-metric space with $s=2$ but not a metric space.

Definition 2.24 Suppose $(Z, \leq)$ is a poset and $T: Z \times Z \rightarrow Z$ be a map. If $T(u, v)$ is monotone nondecreasing in first argument i.e. $u$ and is monotone nonincreasing in second argument i.e. $v$, that is, for all $p, q \in Z, p \leq q$ implies $T(p, v) \leq T(q, v)$ for any $v \in Z$ and for all $x, y \in Z, y \leq x$ implies $T(u, x) \leq T(u, y)$ for any $u \in Z$, then one can say that $\mathrm{T}$ has mixed monotone property .

Definition 2.25 Suppose $(Z, \leq)$ is a poset and $T: Z \times Z \rightarrow Z$ and $g: Z \rightarrow Z$ be two mappings. $\mathrm{T}$ has the mixed g-monotone property if for any $p, q \in Z$, $g p \leq g q$ implies $T(p, y) \leq T(q, y)$ for any $y \in Z$ and for any $u, v \in Z, g u \leq g v$ implies $T(z, v) \leq T(z, u)$ for any $z \in Z$.

Definition 2.26 Suppose $(Z, d, E)$ be E-b-metric space. An element $\left(z_{1}, z_{2}\right) \in Z \times Z$ is said to be coupled fixed point of a function $T: Z \times Z \rightarrow Z$ if $T\left(z_{1}, z_{2}\right)=z_{1}$ and $T\left(z_{2}, z_{1}\right)=z_{2}$.

Definition 2.27 An element of $\left(z_{1}, z_{2}\right) \in Z \times Z$ is said to be coupled coincidence point of the mapping $T: Z \times Z \rightarrow Z$ and $g: Z \rightarrow Z$ if $T\left(z_{1}, z_{2}\right)=g\left(z_{1}\right)=z_{1}$ and $T\left(z_{2}, z_{1}\right)=g\left(z_{2}\right)=z_{2}$.
Definition 2.28 Suppose $Z$ is a non empty set. The mapping $T: Z \times Z \rightarrow Z$ and $g: Z \rightarrow Z$ are said to be commutative

if $g\left(T\left(z_{1}, z_{2}\right)\right)=T\left(g\left(z_{1}\right), g\left(z_{2}\right)\right) \forall x, y \in Z$.

- Definition 2.29 Suppose $Z \neq \phi$ and $T: Z \times Z \rightarrow Z$ and $g: Z \rightarrow Z$. The pair $(T, g)$ is said to be weakly compatible if $g\left(T\left(z_{1}, z_{2}\right)\right)=T\left(g\left(z_{1}\right), g\left(z_{2}\right)\right) \quad$ whenever $g\left(z_{1}\right)=T\left(z_{1}, z_{2}\right)$ and $g\left(z_{2}\right)=T\left(z_{2}, z_{1}\right) \forall z_{1}, z_{2} \in Z \times Z$.

\section{MAIN RESULTS}

Theorem 3.1 Suppose $\left(Z, \leq_{Z}\right)$ is a poset and $d: Z \times Z \rightarrow E_{+}$be E-b-metric defined on $\mathrm{Z}$ with coefficient $s \geq 1$ and E-Archimedean. Let $T: Z \times Z \rightarrow Z$ and $g: Z \rightarrow Z$ be two mappings such that $d(T(u, v), T(p, q))+d(T(v, u), T(q, p)) \leq_{E} k[d(g u, g p)+d(g v, g q)]$ for some $k \in\left(0, \frac{1}{s}\right)$ and for all $u, v, p, q \in Z$ with $g p \leq_{Z} g u$ and $g v \leq_{Z} g q$. We further assume the following hypothesis

(1) $T(Z \times Z) \subseteq g(Z)$

(2) $g(Z)$ is E-complete.

(3) $g$ is vectorially continuous and commute with T.

(4) T has the mixed $g$ - monotone property on Z.

(5)Either T is vectorially continuous or

A. for every non decreasing sequence, if $\left\{u_{n}\right\} \rightarrow u$ then $u_{n} \leq_{Z} u$.

B. for every increasing sequence if $\left\{v_{n}\right\} \rightarrow v$ then $v \leq_{Z} v_{n}$.

Then if there exists two elements $u_{0}, v_{0} \in Z$ with $g\left(u_{0}\right) \leq_{Z} T\left(u_{0}, v_{0}\right)$ and $T\left(u_{0}, v_{0}\right) \leq_{Z} g\left(v_{0}\right), \mathrm{T}$ and $\mathrm{g}$ have coupled coincident fixed point.

Proof: - Let $u_{0}, v_{0} \in Z$ be such that $g u_{0} \leq_{Z} T\left(u_{0}, v_{0}\right)$ and $T\left(v_{0}, u_{0}\right) \leq_{Z} g v_{0}$.

Since $T(Z \times Z) \subseteq g(Z)$, it is to find $u_{1}, v_{1} \in Z$ such that 


\section{Coupled Fixed Point Theorems in Vector b-metric Space}

$$
\begin{aligned}
& \qquad \begin{array}{l}
g\left(u_{1}\right)=T\left(u_{0}, v_{0}\right) \\
\text { and } g\left(v_{1}\right)=T\left(v_{0}, u_{0}\right)
\end{array}
\end{aligned}
$$

Again since $T(Z \times Z) \subseteq g(Z)$, we can select $u_{2}, v_{2} \in Z$ such that

$$
\begin{aligned}
& g\left(u_{2}\right)=T\left(u_{1}, v_{1}\right) \\
& g\left(v_{2}\right)=T\left(v_{1}, u_{1}\right) .
\end{aligned}
$$

and

Continuing this process, we can construct two sequences $\left\{u_{n}\right\}$ and $\left\{v_{n}\right\}$ in $\mathrm{Z}$ such that,

$$
\begin{aligned}
g\left(u_{n+1}\right)= & T\left(u_{n}, v_{n}\right) \\
& \text { and } \\
g\left(v_{n+1}\right)= & T\left(v_{n}, u_{n}\right), \quad \forall n
\end{aligned}
$$

Now we will prove that $\forall n \geq 0$,

$$
\begin{aligned}
& g\left(u_{n}\right) \leq_{Z} g\left(u_{n+1}\right) \\
& g\left(v_{n+1}\right) \leq_{Z} g\left(v_{n}\right)
\end{aligned}
$$

We will prove (3) and (4) by the use of principle mathematical induction.

Suppose $n=0$.

Since $g\left(u_{0}\right) \leq_{Z} T\left(u_{0}, v_{0}\right)$ and $T\left(v_{0}, u_{0}\right) \leq_{Z} g\left(v_{0}\right)$.

Thus we have $g\left(u_{0}\right) \leq_{Z} g\left(u_{1}\right)$ and $g\left(v_{1}\right) \leq_{Z} g\left(v_{0}\right)$.

So (3) and (4) hold for $n=0$.

Now we suppose that (3) and (4) hold for some $n>0$.

Again

$$
\begin{aligned}
& d\left(g u_{n}, g u_{n+1}\right)+d\left(g v_{n}, g v_{n+1}\right)=d\left(T\left(u_{n-1}, v_{n-1}\right), T\left(u_{n}, v_{n}\right)\right)+d\left(T\left(v_{n-1}, u_{n-1}\right), T\left(v_{n}, u_{n}\right)\right) \\
& \leq_{E} k\left[d\left(g u_{n-1}, g u_{n}\right)+d\left(g v_{n-1}, g v_{n}\right)\right]
\end{aligned}
$$

Let $d\left(g u_{n}, g u_{n+1}\right)+d\left(g v_{n}, g v_{n+1}\right)=d_{n}$

where $d_{n}$ is some element of $\mathrm{E}$.

Then $d_{n} \leq_{E} k d_{n-1}$

$\Rightarrow d_{n} \leq_{E} k d_{n-1} \leq_{E} k^{2} d_{n-2} \leq_{E}$ $\leq_{E} k^{n} d_{0}$

Again let $m, n$ be two positive integers, such that $m>n$. Then we can write

$$
\begin{gathered}
d\left(g u_{n}, g u_{m}\right) \leq_{E} s d\left(g u_{n}, g u_{n+1}\right)+s^{2} d\left(g u_{n+1}, g u_{n+2}\right)+s^{3} d\left(g u_{n+2}, g u_{n+3}\right)+\ldots \ldots . . \\
\ldots \ldots \ldots \ldots+s^{m-n-1} d\left(g u_{m-2}, g u_{m-1}\right)+s^{m-n-1} d\left(g u_{m-1}, g u_{m}\right)
\end{gathered}
$$

(By repeated use of triangular inequality)

$$
\begin{gathered}
d\left(g v_{n}, g v_{m}\right) \leq_{E} s d\left(g v_{n}, g v_{n+1}\right)+s^{2} d\left(g v_{n+1}, g v_{n+2}\right)+s^{3} d\left(g v_{n+2}, g v_{n+3}\right)+\ldots \\
\ldots . .+s^{m-n-1} d\left(g v_{m-2}, g v_{m-1}\right)+s^{m-n-1} d\left(g v_{m-1}, g v_{m}\right)
\end{gathered}
$$

Therefore,

$$
d\left(g u_{n}, g u_{m}\right)+d\left(g v_{n}, g v_{m}\right) \leq_{E} s d_{n}+s^{2} d_{n+1}+s^{3} d_{n+2}+\ldots \ldots . .+s^{m-n-1} d_{m-2}+s^{m-n-1} d_{m-1}
$$

\section{[using (7)]}




$$
\begin{aligned}
& \leq_{E} s k^{n} d_{0}+s^{2} k^{n+1} d_{0}+s^{3} k^{n+2} d_{0}+\ldots \ldots .+s^{m-n-1} k^{m-2} d_{0}+s^{m-n-1} k^{m-1} d_{0} \\
& \leq_{E} s k^{n} d_{0}+s^{2} k^{n+1} d_{0}+s^{3} k^{n+2} d_{0}+\ldots \ldots . .+s^{m-n-1} k^{m-2} d_{0}+s^{m-n} k^{m-1} d_{0} \\
& \leq_{E} s k^{n} d_{0}\left[1+s k+s^{2} k^{2}+\ldots \ldots .+s^{m-n-1} k^{m-n-1}\right] \\
& \leq_{E} s k^{n} d_{0}\left[1+s k+s^{2} k^{2}+\ldots \ldots \ldots . .\right] \\
& \leq_{E} s k^{n} d_{0} \frac{1}{1-s k}
\end{aligned}
$$

Thus $d\left(g u_{n}, g u_{m}\right)+d\left(g v_{n}, g v_{m}\right)_{E} \leq 0$

$$
\Rightarrow d\left(g u_{n}, g u_{m}\right) \leq_{E} 0, d\left(g v_{n}, g v_{m}\right) \leq_{E} 0
$$

Hence $\left\{g u_{n}\right\}$ and $\left\{g v_{n}\right\}$ are two E-Cauchy sequence in $g Z$ and we supposed the hypothesis that $g Z$ is E-complete. So there exists two points, say $\mathrm{u}$ and $\mathrm{v}$ in $\mathrm{Z}$, such that the two E-Cauchy sequences $g u_{n} \rightarrow g u=\xi \quad$ and $g v_{n} \rightarrow g v=\eta$ as $n \rightarrow \infty$.

Now let (5) holds, $\mathrm{T}$ is vectorially continuous and so $g\left(g\left(u_{n+1}\right)\right)=g\left(T\left(u_{n}, v_{n}\right)\right)=T\left(g u_{n}, g v_{n}\right)$ since $\mathrm{T}$ and $g$ are commutative.

$\Rightarrow g(\xi)=T(\xi, \eta)$, since $\mathrm{T}$ and $\mathrm{g}$ are vectorially continuous.

Similarly, we can show that $g(\eta)=T(\eta, \xi)$.

Hence $(\xi, \eta)$ is a point of coincidence for $\mathrm{T}$ and $\mathrm{g}$.
Again, let (5A) hold, by (5) we get that $\left\{g u_{n}\right\}$ is a non-decreasing sequence and $g u_{n} \rightarrow \xi$, therefore $g u_{n} \leq_{Z} \xi$, for all $\mathrm{n}$.

Similarly by (5B) and (6), we get that $\left\{g v_{n}\right\}$ is a non-increasing sequence and $g v_{n} \rightarrow \eta$, therefore $\eta \leq_{Z} g v_{n}$, for all $\mathrm{n}$.

Then

$$
\begin{aligned}
& d(g \xi, T(\xi, \eta)) \leq_{E} s d\left(g \xi, g g u_{n+1}\right)+s d\left(g g u_{n+1}, T(\xi, \eta)\right) \\
& =s d\left(g \xi, g g u_{n+1}\right)+s d\left(g\left(T\left(u_{n}, v_{n}\right)\right), T(\xi, \eta)\right) \\
& =s d\left(g \xi, g g u_{n+1}\right)+s d\left(T\left(g u_{n}, g v_{n}\right), T(\xi, \eta)\right)
\end{aligned}
$$

Add both side $s d\left(T\left(g v_{n}, g u_{n}\right), T(\eta, \xi)\right)$, thus we have

$$
\begin{aligned}
& d(g \xi, T(\xi, \eta))+s d\left(T\left(g v_{n}, g u_{n}\right), T(\eta, \xi)\right) \leq_{E} s d\left(g \xi, g g u_{n+1}\right) \\
& +s d\left(T\left(g u_{n}, g v_{n}\right), T(\xi, \eta)\right)+s d\left(T\left(g v_{n}, g u_{n}\right), T(\eta, \xi)\right) \\
& d(g \xi, T(\xi, \eta))+s d\left(T\left(g v_{n}, g u_{n}\right), T(\eta, \xi)\right) \leq_{E} s d\left(g \xi, g g u_{n+1}\right)+s k\left[d\left(g g u_{n}, g \xi\right)+d\left(g g v_{n}, g \eta\right)\right]
\end{aligned}
$$

Since, $\mathrm{g}$ is vectorially E-continuous, $g g u_{n} \rightarrow g \xi$ and $g g v_{n} \rightarrow g \eta \quad$ as $\quad n \rightarrow \infty \quad$ and hence gives $g \xi=T(\xi, \eta)$.

Similarly, we can show that, $g \eta=T(\eta, \xi)$

Again, $d(g \xi, g \eta)+d(g \eta, g \xi)$

$=d(T(\xi, \eta), T(\eta, \xi))+d(T(\eta, \xi), T(\xi, \eta))$

$\leq_{E} k[d(g \xi, g \eta)+d(g \eta, g \xi)]$

$\Rightarrow 2 d(g \xi, g \eta) \leq_{E} 2 k d(g \xi, g \eta)$

$\Rightarrow d(g \xi, g \eta) \leq_{E} k d(g \xi, g \eta)$

Since $k<\frac{1}{s}, \quad d(g \xi, g \eta)=0$, thus $g \xi=g \eta$.

Hence $T(\xi, \eta)=g \xi=g \eta=T(\eta, \xi)$

Finally,

$$
\begin{aligned}
d(\xi, g \xi) & \leq_{E} s d\left(\xi, g u_{n+1}\right)+s d\left(g u_{n+1}, g \xi\right) \\
& =s d\left(\xi, g u_{n+1}\right)+s d\left(T\left(u_{n}, v_{n}\right), T(\xi, \eta)\right)
\end{aligned}
$$

and in the same manner

$$
\begin{aligned}
d(\eta, g \eta) & \leq_{E} s d\left(\eta, g v_{n+1}\right)+s d\left(g v_{n+1}, g \eta\right) \\
& =s d\left(\eta, g v_{n+1}\right)+s d\left(T\left(v_{n}, u_{n}\right), T(\eta, \xi)\right)
\end{aligned}
$$

Therefore,

$$
\begin{aligned}
& d(\xi, g \xi)+d(\eta, g \eta) \leq_{E} s\left[d\left(\xi, g u_{n+1}\right)\right]+d\left(\eta, g v_{n+1}\right) \\
& +s\left[d\left(T\left(u_{n}, v_{n}\right), T(\xi, \eta)\right)+d\left(T\left(v_{n}, u_{n}\right), T(\eta, \xi)\right)\right] \\
& \leq_{E} s\left[d\left(\xi, g u_{n+1}\right)\right]+d\left(\eta, g v_{n+1}\right)+s k\left[d\left(g u_{n}, g \xi\right)+d\left(g v_{n}, g \eta\right)\right] \\
& \leq_{E} s\left[d\left(\xi, g u_{n+1}\right)\right]+d\left(\eta, g v_{n+1}\right)+s^{2} k\left[d\left(g u_{n}, \xi\right)+d(\xi, g \xi)+d\left(g v_{n}, \eta\right)+d(\eta, g \eta)\right]
\end{aligned}
$$




\section{Coupled Fixed Point Theorems in Vector b-metric Space}

Thus $\left(1-k s^{2}\right)[d(\xi, g \xi)+d(\eta, g \eta)]$

(i) $g(Z)$ is E-complete subspace of $Z$.

$\leq_{E} s\left[d\left(\xi, g u_{n+1}\right)+d\left(\eta, g v_{n+1}\right)\right]+s^{2} k\left[d\left(g u_{n}, \xi\right)+d\left(g v_{n}, \eta\right)\right] \rightarrow 0$

as $n \rightarrow \infty$

Thus $d(\xi, g \xi)=0=d(\eta, g \eta)$

$\Rightarrow \xi=g \xi$ and $\eta=g \eta$

$\Rightarrow g(\xi)=\xi=T(\xi, \eta), g(\eta)=\eta=T(\eta, \xi)$

(ii) $T(Z \times Z) \subseteq g(Z)$

(iii) $s\left(t_{1}+t_{2}+t_{3}+t_{4}\right)+t_{5}+t_{6}+t_{7}+t_{8}+\left(s^{2}+s\right)\left(t_{9}+t_{10}\right)<1$

Then $\mathrm{T}$ and $\mathrm{g}$ have coupled coincidence point. Further, if $\mathrm{T}$ and $g$ are weakly compatible then $\mathrm{T}$ and $\mathrm{g}$ have unique coupled fixed point.

Proof: - Take $v_{0} \in Z$.

Theorem 3.2 Suppose $(Z, d, E)$ is a E-b-metric space with $s>1, \quad$ E-Archimedean and $T: Z \times Z \rightarrow Z$ and $g: Z \rightarrow Z$ be two mappings on $Z$. Suppose that there exists non-negative constant $t_{i}, i=1,2, \ldots, 10$ such that

$d(T(u, v), T(p, q)) \leq_{E} t_{1} d(g u, g p)+t_{2} d(g v, g q)+t_{3} d(T(u, v), g u)+t_{4} d(T(v, u), g v)$ $+t_{5} d(T(p, q), g p)+t_{6} d(T(q, p), g q)+t_{7} d(T(u, v), g p)+t_{8} d(T(v, u), g q)$ $+t_{9} d(T(p, q), g u)+t_{10} d(T(q, p), g v)$

Since $T(Z \times Z) \subseteq g(Z)$, then can find $v_{1} \in Z$ such that $T\left(v_{0}, v_{0}\right)=g\left(v_{1}\right)$

Again since $T(Z \times Z) \subseteq g(Z)$, then $\exists v_{2} \in Z$ such that $T\left(v_{1}, v_{1}\right)=g\left(v_{2}\right)$.

Repeating the above process, we will get a sequence $\left\{u_{n}\right\}$ in $g(Z)$ such that

holds for all $u, v, p, q \in Z$.

$$
u_{n}=g\left(v_{n+1}\right)=T\left(v_{n}, v_{n}\right)
$$

Suppose

$$
\begin{aligned}
& d\left(g v_{n+1}, g v_{n+2}\right)=d\left(T\left(v_{n}, v_{n}\right), T\left(v_{n+1}, v_{n+1}\right)\right) \leq_{E} t_{1} d\left(g v_{n}, g v_{n+1}\right)+t_{2} d\left(g v_{n}, g v_{n+1}\right) \\
& +t_{3} d\left(T\left(v_{n}, v_{n}\right), g v_{n}\right)+t_{4} d\left(T\left(v_{n}, v_{n}\right), g v_{n}\right)+t_{5} d\left(T\left(v_{n+1}, v_{n+1}\right), g v_{n}\right) \\
& +t_{6} d\left(T\left(v_{n+1}, v_{n+1}\right), g v_{n+1}\right)+t_{7} d\left(T\left(v_{n}, v_{n}\right), g v_{n+1}\right)+t_{8} d\left(T\left(v_{n}, v_{n}\right), g v_{n+1}\right) \\
& +t_{9} d\left(T\left(v_{n+1}, v_{n+1}\right), g v_{n}\right)+t_{10} d\left(T\left(v_{n+1}, v_{n+1}\right), g v_{n}\right)
\end{aligned}
$$

by using (9), we have

$$
\begin{aligned}
& \Rightarrow d\left(g v_{n+1}, g v_{n+2}\right) \leq_{E} t_{1} d\left(g v_{n}, g v_{n+1}\right)+t_{2} d\left(g v_{n}, g v_{n+1}\right)+t_{3} d\left(g v_{n+1}, g v_{n}\right)+t_{4} d\left(g v_{n+1}, g v_{n}\right) \\
& +t_{5} d\left(g v_{n+2}, g v_{n+1}\right)+t_{6} d\left(g v_{n+2}, g v_{n+1}\right)+t_{7} d\left(g v_{n+1}, g v_{n+1}\right) \\
& +t_{8} d\left(g v_{n+1}, g v_{n+1}\right)+t_{9} d\left(g v_{n+2}, g v_{n}\right)+t_{10} d\left(g v_{n+2}, g v_{n}\right) \\
& \left(1-t_{5}-t_{6}\right) d\left(g v_{n+1}, g v_{n+2}\right) \leq_{E}\left(t_{1}+t_{2}+t_{3}+t_{4}\right) d\left(g v_{n}, g v_{n+1}\right)+\left(t_{9}+t_{10}\right) d\left(g v_{n+2}, g v_{n}\right)
\end{aligned}
$$

Since, $\quad d\left(g v_{n}, g v_{n+2}\right) \leq_{E} s d\left(g v_{n}, g v_{n+1}\right)+s d\left(g v_{n+1}, g v_{n+2}\right)$

$$
\begin{aligned}
& \begin{array}{l}
\left(1-t_{5}-t_{6}\right) d\left(g v_{n+1}, g v_{n+2}\right) \leq_{E}\left(t_{1}+t_{2}+t_{3}+t_{4}\right) d\left(g v_{n}, g v_{n+1}\right) \\
\quad \\
\quad+s\left(t_{9}+t_{10}\right)\left[d\left(g v_{n}, g v_{n+1}\right)+d\left(g v_{n+1}, g v_{n+2}\right)\right]
\end{array} \\
& \begin{array}{r}
\Rightarrow\left(1-t_{5}-t_{6}-s\left(t_{9}+t_{10}\right)\right) d\left(g v_{n+1}, g v_{n+2}\right) \leq_{E}\left(t_{1}+t_{2}+t_{3}+t_{4}+s\left(t_{9}+t_{10}\right)\right) d\left(g v_{n}, g v_{n+1}\right) \\
\Rightarrow d\left(g v_{n+1}, g v_{n+2}\right) \leq_{E} \frac{t_{1}+t_{2}+t_{3}+t_{4}+s\left(t_{9}+t_{10}\right)}{1-t_{5}-t_{6}-s\left(t_{9}+t_{10}\right)} d\left(g v_{n}, g v_{n+1}\right)
\end{array} \\
& \begin{array}{l}
\Rightarrow \\
\text { where } r=\frac{t_{1}+t_{2}+t_{3}+t_{4}+s\left(t_{9}+t_{10}\right)}{1-t_{5}-t_{6}-s\left(t_{9}+t_{10}\right)}
\end{array} \\
& \text { sr }=\frac{s\left(t_{1}+t_{2}+t_{3}+t_{4}\right)+s^{2}\left(t_{9}+t_{10}\right)}{1-t_{5}-t_{6}-s\left(t_{9}+t_{10}\right)} \\
& \text { Since } s\left(t_{1}+t_{2}+t_{3}+t_{4}\right)+t_{5}+t_{6}+\left(s^{2}+s\right)\left(t_{9}+t_{10}\right)<1
\end{aligned}
$$


By using the condition (i),

$s\left(t_{1}+t_{2}+t_{3}+t_{4}\right)+t_{5}+t_{6}+t_{7}+t_{8}+\left(s^{2}+s\right)\left(t_{9}+t_{10}\right)<1$ and $r \leq s r$, we get $r<1$,

$\Rightarrow d\left(g v_{n+1}, g v_{n+2}\right) \leq_{E} r d\left(g v_{n}, g v_{n+1}\right)$

Repeating n-times,

$d\left(g v_{n+1}, g v_{n+2}\right) \leq_{E} r^{n+1} d\left(g v_{0}, g v_{1}\right)$

Let $n, m \in N, m>n$.

Therefore

$$
\begin{array}{rl}
d\left(g v_{n}, g v_{m}\right) \leq_{E} & s d\left(g v_{n}, g v_{n+1}\right)+s^{2} d\left(g v_{n+1}, g v_{n+2}\right)+\ldots \ldots \ldots . . .+s^{m-n} d\left(g v_{m-1}, g v_{m}\right) \\
\Rightarrow d\left(g v_{n}, g v_{m}\right) & \leq_{E} s r^{n} d\left(g v_{0}, g v_{1}\right)+s^{2} r^{n+1} d\left(g v_{0}, g v_{1}\right)+\ldots \ldots \ldots . . .+s^{m-n} r^{m-1} d \\
& =s r^{n} d\left(g v_{0}, g v_{1}\right)\left[1+s r+s r^{2}+\ldots \ldots \ldots . s^{m-n-1} r^{m-n-1}\right] \\
& \leq_{E} s r^{n} d\left(g v_{0}, g v_{1}\right)\left[\frac{1}{1-s r}\right]
\end{array}
$$

Since

$r<1 \Rightarrow r^{n} \rightarrow 0$ as $n \rightarrow \infty$,

$s r<1 \Rightarrow(1-s r)>0$

$\Rightarrow\left\{g v_{n}\right\}$ is an E-Cauchy sequence in $(g(Z), d)$.

Since $g(Z)$ is complete, $\exists t \in g(Z)$ such that

$$
\lim _{n \rightarrow \infty} g\left(v_{n}\right)=g(v)=t
$$

We will now prove that $(y, y)$ is the coupled coincident point of $\mathrm{T}$ and $\mathrm{g}$.

$$
\begin{aligned}
d\left(g v_{n+1}, T(v, v)\right)= & d\left(T\left(v_{n}, v_{n}\right), T(v, v)\right) \\
& \leq_{E} t_{1} d\left(g v_{n}, g v\right)+t_{2} d\left(g v_{n}, g v\right)+t_{3} d\left(T\left(v_{n}, v_{n}\right), g v_{n}\right)+t_{4} d\left(T\left(v_{n}, v_{n}\right), g v_{n}\right) \\
& +t_{5} d(T(v, v), g v)+t_{6} d(T(v, v), g v)+t_{7} d\left(T\left(v_{n}, v_{n}\right), g v\right)+t_{8} d\left(T\left(v_{n}, v_{n}\right), g v\right) \\
& +t_{9} d\left(T(v, v), g v_{n}\right)+t_{10} d\left(T(v, v), g v_{n}\right) \\
d\left(g v_{n+1}, T(v, v)\right)= & d\left(T\left(v_{n}, v_{n}\right), T(v, v)\right) \\
& \leq_{E} t_{1} d\left(g v_{n}, g v\right)+t_{2} d\left(g v_{n}, g v\right)+t_{3} d\left(g v_{n+1}, g v_{n}\right)+t_{4} d\left(g v_{n+1}, g v_{n}\right) \\
& +t_{5} d(T(v, v), g v)+t_{6} d(T(v, v), g v)+t_{7} d\left(g v_{n+1}, g v\right)+t_{8} d\left(g v_{n+1}, g v\right) \\
& +t_{9} d\left(T(v, v), g v_{n}\right)+t_{10} d\left(T(v, v), g v_{n}\right)
\end{aligned}
$$

Since $\quad d(g v, T(v, v)) \leq_{E} s\left[d\left(g v, g v_{n+1}\right)+d\left(g v_{n+1}, T(v, v)\right)\right]$

$\Rightarrow \frac{1}{s} d(g v, T(v, v)) \leq_{E} \lim _{n \rightarrow \infty} d\left(g v_{n+1}, T(v, v)\right)$

Also, $\quad d\left(g v_{n+1}, g v_{n}\right) \leq_{E} s\left[d\left(g v_{n+1}, g v\right)+d\left(g v, g v_{n}\right)\right]$

Thus $\lim d\left(g v_{n+1}, g v_{n}\right)=0$

Further,

$d\left(T(v, v), g v_{n}\right) \leq_{E} s\left[d(T(v, v), g v)+d\left(g v, g v_{n}\right)\right]$

Letting $n \rightarrow \infty$ in above inequality,

$\lim _{n \rightarrow \infty} d\left(T(v, v), g v_{n}\right) \leq_{E} s d(T(v, v), g v)$

Taking $\lim n \rightarrow \infty$ in (12) and using (15) 


\section{Coupled Fixed Point Theorems in Vector b-metric Space}

$$
\begin{aligned}
& \frac{1}{s} d(g v, T(v, v)) \leq_{E} t_{5} d(T(v, v), g v)+t_{6} d(T(v, v), g v)+s t_{9} d(T(v, v), g v)+s t_{10} d(T(v, v), g v) \\
& \Rightarrow d(g v, T(v, v)) \leq_{E}\left(s t_{5}+s t_{6}+s^{2} t_{9}+s^{2} t_{10}\right) d(T(v, v), g v)
\end{aligned}
$$

Since $s t_{5}+s t_{6}+s^{2} t_{9}+s^{2} t_{10}<1 \quad$ Using condition (i)

and $\mathrm{E}$ is Archimedean

$\Rightarrow g(v)=T(v, v)$

$\Rightarrow(v, v)$ is a coupled coincidence point of $\mathrm{T}$ and $\mathrm{g}$.

For uniqueness of couple coincidence point $(v, v)$, suppose $\left(v^{\prime}, v^{\prime}\right)$ be another coupled coincidence point of $\mathrm{T}$ and $\mathrm{g}$. Then

$$
\begin{aligned}
d\left(g v, g v^{\prime}\right)= & d\left(T(v, v), T\left(v^{\prime}, v^{\prime}\right)\right) \\
& \leq_{E} t_{1} d\left(g v, g v^{\prime}\right)+t_{2} d\left(g v, g v^{\prime}\right)+t_{3} d(T(v, v), g v)+t_{4} d(T(v, v), g v) \\
& +t_{5} d\left(T\left(v^{\prime}, v^{\prime}\right), g v\right)+t_{6} d\left(T\left(v^{\prime}, v^{\prime}\right), g v^{\prime}\right)+t_{7} d\left(T(v, v), g v^{\prime}\right) \\
& +t_{8} d\left(T(v, v), g v^{\prime}\right)+t_{9} d\left(T\left(v^{\prime}, v^{\prime}\right), g v\right)+t_{10} d\left(T\left(v^{\prime}, y^{\prime}\right), g v\right) \\
d\left(g v, g v^{\prime}\right) \leq & \left(t_{1}+t_{2}+t_{7}+t_{8}+t_{9}+t_{10}\right) d\left(g v, g v^{\prime}\right)
\end{aligned}
$$

Since $t_{1}+t_{2}+t_{7}+t_{8}+t_{9}+t_{10}<1$

$$
\Rightarrow g(v)=g\left(v^{\prime}\right)
$$

Hence $(v, v)$ is the unique coupled coincidence point of $\mathrm{T}$ and $\mathrm{g}$.

Since $\mathrm{T}$ and $\mathrm{g}$ are weakly compatible, then

$$
\Rightarrow g(T(v, v))=T(g v, g v)
$$

Put $w=g(v)$

$g(w)=g(g v)=g(T(v, v))=T(g v, g v)$

$\Rightarrow(w, w)$ is coupled coincidence point of $\mathrm{T}$ and $\mathrm{g}$.

By uniqueness of coupled coincidence point of $\mathrm{T}$ and $\mathrm{g}, w=v$ i.e. $g(y)=v$

But $T(v, v)=g v=v$

$\Rightarrow(v, v)$ is coupled fixed point of $\mathrm{T}$ and $\mathrm{g}$.

Corollary 3.3 Let $(Z, d, E)$ be Archimedean E-b-metric space with constant $s \geq 1$ and E-Complete. Let $T: Z \times Z \rightarrow Z$ be a mapping. Suppose there exist non-negative constants $t_{i}, 1 \leq i \leq 10, i \in \square$ such that

$$
\begin{aligned}
d(T(u, v), T(p, q)) & \leq_{E} t_{1} d(u, p)+t_{2} d(v, q)+t_{3} d(T(u, y), u) \\
& +t_{4} d(T(v, u), v)+t_{5} d(T(p, q), p) \\
& +t_{6} d(T(q, p), q)+t_{7} d(T(u, v), p) \\
& +t_{8} d(T(v, u), q)+t_{9} d(T(p, q), u)+t_{10} d(T(q, p), v)
\end{aligned}
$$

holds for all $u, v, p, q \in Z$.

If $s\left(\sum_{i=1}^{6} t_{i}\right)+t_{7}+t_{8}+\left(s^{2}+s\right) t_{9}+\left(s^{2}+s\right) t_{10}<1$, then $\mathrm{T}$ has a unique coupled fixed point.

Proof: Simply take g=I (identity) in theorem 3.2.and repeat the above proof.

Corollary 3.4 Let $(Z, d, E)$ be Archimedean E-b-metric space with constant $s \geq 1$ and E-Complete. Let $T: Z \times Z \rightarrow Z$ be a mapping. Suppose $\exists t_{i}, i=1,2, \ldots, 10$ where

$t_{i} \geq 0$ such that 


$$
\begin{aligned}
d(T(u, v), T(p, q)) & \leq_{E} t_{1} d(u, p)+t_{2} d(v, q)+t_{3} d(T(u, v), u) \\
& +t_{4} d(T(v, u), v)+t_{5} d(T(p, q), p) \\
& +t_{6} d(T(q, p), q)+t_{7} d(T(u, v), p) \\
& +t_{8} d(T(v, u), q)+t_{9} d(T(p, q), u)+t_{10} d(T(q, p), v)
\end{aligned}
$$

holds $\forall u, v, p, q \in Z$.

If $\sum_{i=1}^{8} t_{i}+2 t_{9}+2 t_{10}<1$, then $\mathrm{T}$ has a unique coupled fixed point.

Proof: Put $s=1$ in the proof of above corollary 3.3 .

Example 3.5 If $Z=\square, E=\square^{2}$, let $d(u, v)=\left(\alpha|u-v|^{2}, \beta|u-v|^{2}\right)$ where $\alpha, \beta \geq 0, \alpha+\beta>0$.

Delineate $T: Z \times Z \rightarrow Z$ and $g: Z \rightarrow Z$ by

$T(u, v)=\frac{u-v}{12}, g(u)=1-\frac{u}{2}$

Then $T(Z \times Z) \subseteq g(Z)=Z$

Then

$$
\begin{aligned}
d(T(u, v), T(p, q)) & =d\left(\frac{u-v}{12}, \frac{p-q}{12}\right) \\
& =\left(\alpha \frac{|u-v-p+q|^{2}}{(12)^{2}}, \beta \frac{|u-v-p+q|^{2}}{(12)^{2}}\right) \\
& \leq\left(\alpha \cdot \frac{\left(|u-p|^{2}+|v-q|^{2}\right)}{(12)^{2}}, \beta \frac{\left(|u-p|^{2}+|v-q|^{2}\right)}{(12)^{2}}\right) \\
& =\frac{2}{72}\left(\alpha \frac{|u-p|^{2}}{2^{2}}+\alpha \frac{|v-q|^{2}}{2^{2}}, \beta \frac{|u-p|^{2}}{2^{2}}+\beta \frac{|v-q|^{2}}{2^{2}}\right) \\
& =\frac{1}{36}[d(g u, g p)+d(g v, g q)]
\end{aligned}
$$

$\Rightarrow t_{1}=t_{2}=\frac{1}{36}, \quad t_{i}=0$ for $i=3,4, \ldots, 10$

$t_{1}+t_{2}=\frac{1}{18}<1$

\section{REFERENCES}

[1] S. Agarwal, K. Qureshi, J.Nema, A fixed point theorem for b-metric space, International Journal of Pure and Applied Mathematical Sciences, $9(2016), 45-50$.

[2] C.D. Aliprantis, K.C. Border, Infinite Dimensional Analysis, Springer-Verlag, Berlin, 1999.

[3] I.A.Bakhtin, The contraction mapping principle in quasi-metric spaces, Functional Analysis, 30(1989), 26-37.

[4] C. Cevik, I. Altun, Vector metric spaces and some properties, Topol. Method Nonl. An., 34(2009), 375-382.

[5] S.Czerwik, Contraction mappings in b-metric spaces, Acta Mathematica et Informatica Universitatis Ostrviensis, 1(1993), 5-11.

[6] I.R.Petre, Fixed point theorems in E-b-metric spaces, J. Nonlinear Sci. App., 7(2014), 264-271.

[7] K.P.R. Rao, K.R.K.Rao, V.C.C.Raju, A Suzuki type unique common coupled fixed point theorem in metric spaces, International Journal of Innovative Research in Science, Engineering and Technology, 2(2013), 5187-5192.

[8] W. Shatanawi, M.B.Hani, A coupled fixed point theorem in b-metric spaces, International Journal of Pure and Applied Mathematics, vol. 19, 4(2016), 889-897.

[9] I.Altun, A.Erduran, A Suzuki type fixed point theorem, Internat. Math. Math. Sci., vol. 2011, article ID 736063, 9 pages.
[10] W. Shatanawi, Coupled fixed point theorems in generalized metric spaces, Hacettepe Journal of Mathematics and Statistics, vol. 40 3(2011), 441-447.

[11] W.Shatanawi, B.Samet, M.Abbas, Coupled fixed point theorems for mixed monotone mappings in ordered partial metric spaces, Mathematical and Computer Modelling, 55(2012), 680-687.

[12] T. Bhakar, V. Lakshmikanthan, Fixed point theorems in partially ordered metric spaces and applications, Nonlinear Anal., 65(2006), 1379-1393.

[13] Y.J. Cho, Z. Kadeelburg, R. Saadati, W.Shatanawi, Coupled fixed theorems under weak contractions, Discrete Dyanamics in Nature and Society (2012).

[14] V. Lakshmikantham, L.B. Ciric , Coupled fixed point theorems for nonlinear contractions in partially ordered metric spaces, Nonlinear Anal., 70 (2009)

[15] I.Altun, C.Cevik, Some common fixed point theorems in vector metric spaces, Filomat, 25:1(2011), 105-113.

[16] C.Cevik, On continuity of functions between vector metric spaces, Hindawi Pub. Corporation, J.Funct. Space, (2014), 1-6. 